\title{
Function Generation Synthesis with Planar 4-bar Linkage as a Mixed Problem of Correlation of Crank Angles and Dead-Center Design
}

\author{
Gökhan Kiper ${ }^{10000-0001-8793-724 X]}$ and Melih Erez $^{2}$ \\ ${ }^{1}$ İzmir Institute of Technology, 35430 İzmir, Turkey \\ ${ }^{2}$ Mecalac İş Makineleri Sanayi ve Ticaret Ltd Şti, 35410 İzmir, Turkey \\ gokhankiper@iyte.edu.tr
}

\begin{abstract}
The function generation synthesis of planar four-bar linkage is formulated as a mixed problem of correlation or crank angles and dead-center design. The synthesis is based on three desired configurations of the linkage, where the input and output joint angles are specified for two of the configurations and the coupler link is folded upon the output link for the third configuration. The formulation results in a set of nonlinear equations which can be solved analytically. The formulations are tested with numerical examples and similar mixed function generation problems are briefly discussed.
\end{abstract}

Keywords: Function Generation Synthesis, Correlation of Crank Angles, DeadCenter Design.

\section{Introduction}

In many applications linkage design problem can be formulated as function generation. In function generation synthesis, the link lengths of a linkage are determined according to a desired relationship between the input and the output joint variables. Formulation of the function generation synthesis problem depends on the whether it is desired that the input and output joint variables should satisfy certain values (precision points) or it is desired that the output joint variable should vary in a given interval while the value of the input joint variable can be continuously varied. For the planar four-bar linkage Söylemez [1] names the first type of formulation as the "correlation of crank angles" whereas the second type is known as "dead-center design". Many classical text books such as [2-4] do not cover the dead-center design problem. Hall [5] and Norton [6] treats the dead-center design and correlation of crank angles problems separately. Mallik et al. [7] presents the dead-center problems as fourth type of synthesis problem in addition to motion, path and function generation problems. Pennestrì and Valentini [8] summarized the analytical function generation synthesis methods for the four-bar and the slider-crank mechanisms for two and three prescribed positions including the deadcenter design problems. According to the author's knowledge, there is publication that treats the correlation or crank angles and dead-center design problems as a mixed synthesis problem. 
In certain applications, a planar four-bar linkage needs to satisfy certain input/output joint values, but also the coupler link becomes collinear with either of the cranks in a limiting position. As an example, for the loader mechanism of a construction machine, the bucket is required to have a certain maximum angle in the lowest and highest positions of the boom. In these two positions, when the boom and actuation cylinders are already designed, the four-bar attached to the bucket need to satisfy two sets of given input and output angles. However, the minimum angle of the bucket at the highest position of the boom is obtained for the dead-center (or near dead-center) position of the four-bar-loop attached to the bucket. This paper presents an analytical solution to such a mixed problem.

\section{Problem Definition and Formulation}

For four-bar function generators since the input and output joint variables are angles, the linkage can be scaled without effecting the input/output relationship, so without loss of generality let fixed link length be 1 unit. For the problem at hand, the input and output joint angles $\phi_{1}, \psi_{1}, \phi_{2}, \psi_{2}$ are specified for position \#1 and position \#2 and only the output angle $\psi_{3}$ is specified for position \#3, where the coupler link is folded upon the output link (Fig. 1). The conventional dead-center design problem is posed in terms of the desired swing-angle between the folded and extended dead-center positions and corresponding crank rotation (or time ratio), but in this case only the folded dead-center position is specified with a given desired output link angle.

Position \#1

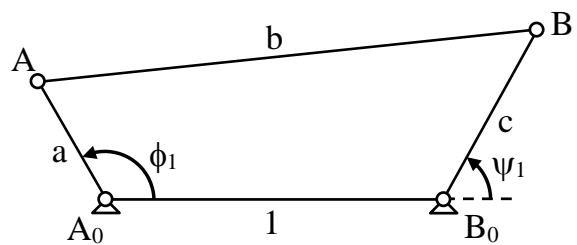

Position \#2

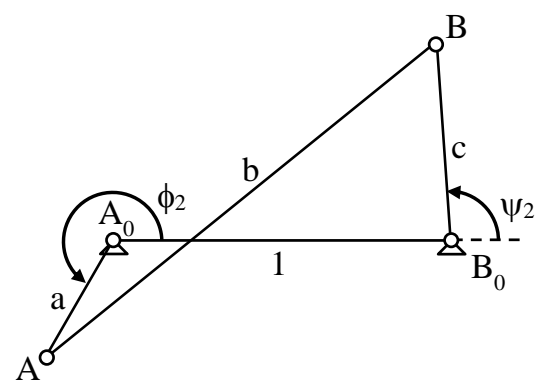

Position \#3

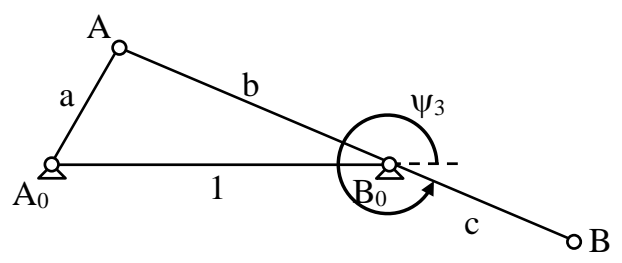

Fig. 1. The three desired positions of a four-bar linkage 
The input/output equation for the four-bar linkage can be derived as follows:

$$
|\overrightarrow{\mathrm{AB}}|=\left|\overrightarrow{\mathrm{A}_{0} \mathrm{~B}_{0}}+\overrightarrow{\mathrm{B}_{0} \mathrm{~B}}-\overrightarrow{\mathrm{A}_{0} \mathrm{~A}}\right| \Rightarrow \mathrm{b}^{2}=(1+\mathrm{c} \cos \psi-\mathrm{a} \cos \phi)^{2}+(\mathrm{c} \sin \psi-\mathrm{a} \sin \phi)^{2}
$$

Expanding Eq. (1) and writing for positions \#1 and \#2:

$$
\begin{aligned}
& 1+\mathrm{a}^{2}-\mathrm{b}^{2}+\mathrm{c}^{2}-2 \mathrm{a} \cos \phi_{1}+2 \mathrm{c} \cos \psi_{1}=2 \mathrm{accos}\left(\phi_{1}-\psi_{1}\right) \\
& 1+\mathrm{a}^{2}-\mathrm{b}^{2}+\mathrm{c}^{2}-2 \mathrm{a} \cos \phi_{2}+2 \mathrm{c} \cos \psi_{2}=2 \mathrm{accos}\left(\phi_{2}-\psi_{2}\right)
\end{aligned}
$$

Writing cosine theorem in triangle $\mathrm{A}_{0} \mathrm{AB}_{0}$ for position \#3 ( $\phi_{3}$ is unknown):

$$
1+(b-c)^{2}-2(b-c) \cos \psi_{3}=a^{2} \Rightarrow 1-a^{2}+(b-c)^{2}=2(b-c) \cos \psi_{3}
$$

For given angle values Eqs. (2)-(4) constitute 3 equation in 3 unknowns (a, b, c). For a correlation of crank angles problem with three positions all equations are alike and the problem can be transformed into a set of linear equations (Freudenstein equations [9]) in newly introduced coefficients. However in this case, Eq. (4) is different from Eqs. (2) and (3). A simple way to solve these equations is to solve for two of the link lengths from Eqs. (2) and (3) and substitute the solution into Eq. (4) to obtain a single equation in single unknown. Let $\lambda=\frac{\mathrm{b}^{2}-1-\mathrm{a}^{2}-\mathrm{c}^{2}}{2 \mathrm{ac}}$. Then

$$
\mathrm{b}^{2}=1+\mathrm{a}^{2}+\mathrm{c}^{2}+2 \mathrm{ac} \lambda
$$

Dividing Eqs. (2) and (3) by 2ac and rearranging:

$$
\begin{aligned}
& \cos \psi_{1} \frac{1}{\mathrm{a}}-\cos \phi_{1} \frac{1}{\mathrm{c}}=\lambda+2 \mathrm{ac} \cos \left(\phi_{1}-\psi_{1}\right) \\
& \cos \psi_{2} \frac{1}{\mathrm{a}}-\cos \phi_{2} \frac{1}{\mathrm{c}}=\lambda+2 \mathrm{ac} \cos \left(\phi_{2}-\psi_{2}\right)
\end{aligned}
$$

Eqs. (6) and (7) are linear in 1/a and 1/c. a and c solved from Eqs. (6) and (7) as

$$
\begin{aligned}
& \mathrm{a}=\frac{\mathrm{f}_{1}}{\mathrm{f}_{2} \lambda+\mathrm{f}_{3}}=\frac{\cos \phi_{1} \cos \psi_{2}-\cos \psi_{1} \cos \phi_{2}}{\left(\cos \phi_{1}-\cos \phi_{2}\right) \lambda+\cos \phi_{1} \cos \left(\phi_{2}-\psi_{2}\right)-\cos \left(\phi_{1}-\psi_{1}\right) \cos \phi_{2}} \\
& \mathrm{c}=\frac{\mathrm{f}_{1}}{\mathrm{f}_{4} \lambda+\mathrm{f}_{5}}=\frac{\cos \phi_{1} \cos \psi_{2}-\cos \psi_{1} \cos \phi_{2}}{\left(\cos \psi_{1}-\cos \psi_{2}\right) \lambda+\cos \psi_{1} \cos \left(\phi_{2}-\psi_{2}\right)-\cos \left(\phi_{1}-\psi_{1}\right) \cos \psi_{2}}
\end{aligned}
$$

Substituting $b^{2}$ from Eq. (5) into Eq. (4):

$$
\begin{gathered}
\mathrm{b}^{2}=1+\mathrm{a}^{2}+\mathrm{c}^{2}+2 \mathrm{ac} \lambda=\mathrm{a}^{2}-1-\mathrm{c}^{2}+2 \mathrm{bc}+2(\mathrm{~b}-\mathrm{c}) \cos \psi_{3} \\
\Rightarrow 1+\mathrm{c}^{2}+\mathrm{ac} \lambda=\mathrm{bc}+(\mathrm{b}-\mathrm{c}) \cos \psi_{3}
\end{gathered}
$$


$\mathrm{b}$ is eliminated by leaving $\mathrm{b}$ alone in Eq. (9), taking square and using Eq. (5) again:

$$
\begin{aligned}
& \left(\mathrm{c}+\cos \psi_{3}\right)^{2} \mathrm{~b}^{2}=\left(\mathrm{c}+\cos \psi_{3}\right)^{2}\left(1+\mathrm{a}^{2}+\mathrm{c}^{2}+2 \mathrm{ac} \lambda\right)=\left(1+\mathrm{c}^{2}+\mathrm{ac} \lambda+\mathrm{c} \cos \psi_{3}\right)^{2} \\
\Rightarrow & \sin ^{2} \psi_{3}+\mathrm{a}^{2} \mathrm{c}^{2}\left(\lambda^{2}-1\right)+\mathrm{c}^{2}+2 \sin ^{2} \psi_{3} \mathrm{ac} \lambda-\cos ^{2} \psi_{3} \mathrm{a}^{2}-2 \cos \psi_{3} \mathrm{ac}(\mathrm{a}+\mathrm{c} \lambda)=0
\end{aligned}
$$

Substituting a and c from Eq. (8) into Eq. (10) and rearranging:

$$
\begin{gathered}
\left(\mathrm{f}_{2} \mathrm{f}_{4} \sin \psi_{3}\right)^{2} \lambda^{4}+2 \mathrm{f}_{2} \mathrm{f}_{4}\left(\mathrm{f}_{1}^{2}+\mathrm{f}_{3} \mathrm{f}_{4}+\mathrm{f}_{2} \mathrm{f}_{5}\right) \sin ^{2} \psi_{3} \lambda^{3} \\
+\left\{\left(\mathrm{f}_{3} \mathrm{f}_{4}+\mathrm{f}_{2} \mathrm{f}_{5}\right)^{2}+2 \mathrm{f}_{2} \mathrm{f}_{3} \mathrm{f}_{4} \mathrm{f}_{5}\right] \sin ^{2} \psi_{3} \\
+2\left\{\mathrm{f}_{3} \mathrm{f}_{5}\left(\mathrm{f}_{3} \mathrm{f}_{4}+\mathrm{f}_{2} \mathrm{f}_{5}\right) \sin ^{2} \psi_{3}+\mathrm{f}_{1}^{2}\left[\left(\mathrm{f}_{2}+\mathrm{f}_{5}\right) \mathrm{f}_{3}-\left(\mathrm{f}_{3}+\mathrm{f}_{4}\right)\left(\mathrm{f}_{1}+\mathrm{f}_{5} \cos \psi_{3}\right) \cos \psi_{3}\right]\right\} \lambda \\
+\left(\mathrm{f}_{3} \mathrm{f}_{5} \sin \psi_{3}\right)^{2}-\mathrm{f}_{1}^{2}\left[\left(\mathrm{f}_{1}+\mathrm{f}_{5} \cos \psi_{3}\right)^{2}-\mathrm{f}_{3}^{2}\right]=0
\end{gathered}
$$

Eq. (11) is a fourth order polynomial in $\lambda$ and has analytical solution with four or two or zero real solutions. For a real $\lambda$ solution the link length values are solved as

$$
\begin{gathered}
a=\frac{f_{1}}{f_{2} \lambda+f_{3}} \\
c=\frac{f_{1}}{f_{4} \lambda+f_{5}} \\
b=c+\frac{1+a c \lambda}{c+\cos \psi_{3}}
\end{gathered}
$$

\section{$3 \quad$ Numerical Simulation}

The formulations in Section 3 are implemented in Microsoft Excel (Fig. 2). Note that all angle values can be negated without effecting the result because the cosine function is an even function and sine function is always squared in the formulations. The negated angle values correspond to the horizontal mirror image of the linkage.

For the specific example in Fig. 2 the prescribed positions are defined by the angles $\phi_{1}=90^{\circ}, \psi_{1}=40^{\circ}, \phi_{2}=140^{\circ}, \psi_{2}=80^{\circ}$ and $\psi_{3}=-20^{\circ}$. There are four real solutions for $\lambda$ in this case. For several worked out examples it is seen that usually there are two real and two complex solutions. For two or four real solutions it is always observed that at most one of the solutions satisfies the desired 3 positions in the same assembly mode of the linkage. The other real solution(s) for $\lambda$ would correspond to solutions with negative link lengths, which are not acceptable. 


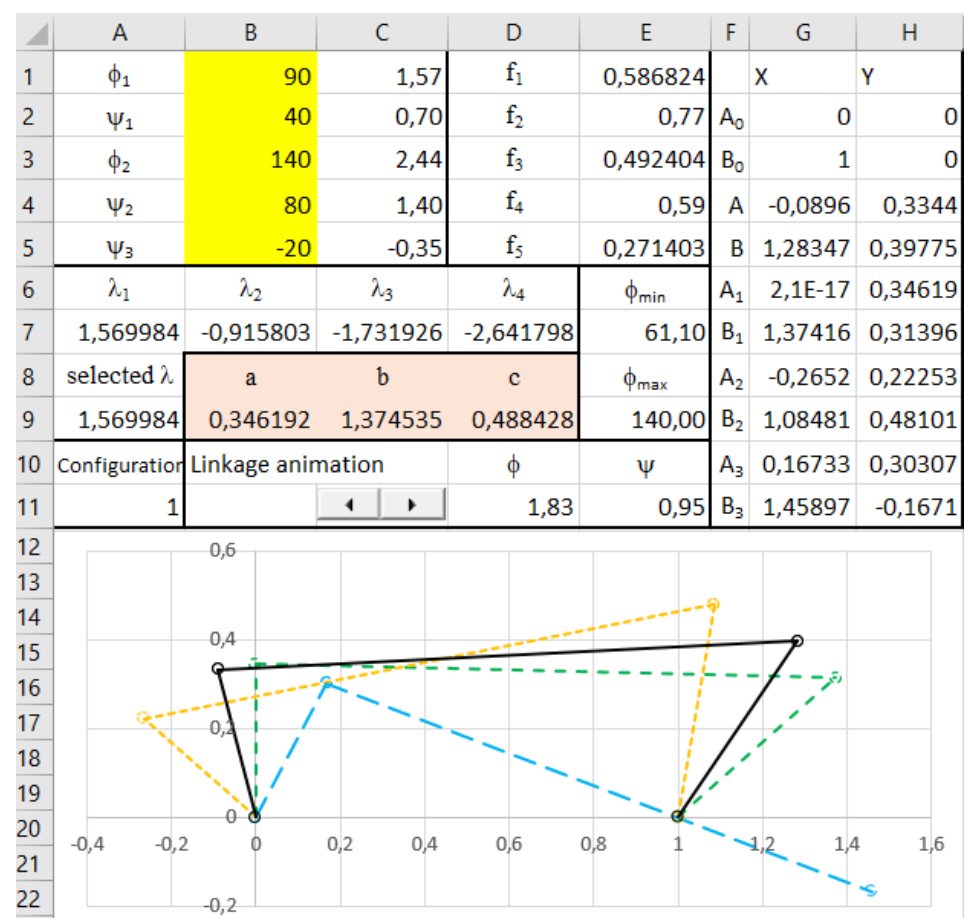

Fig. 2. Numerical implementation in Excel

\section{Discussion}

This paper presents formulations for the synthesis of a planar four-bar linkage for given two sets of input and output joint variables and a limiting position where the coupler link is folded upon the output link with a given output angle. Such a problem differs from the conventional correlation of crank angles type function generation problem in the sense that not all the equations have the same form. The solution of such a mixed function generation problem can easily be found using a CAD software, but when the four-bar design is merely a part of the design of a multi-loop mechanism, it is necessary to implement solution of a parametric model and an analytical solution is required for immediate computation. This paper presents such an analytical formulation.

Similar formulations can be easily derived for extended case instead of the folded case (just use $b+c$ instead of $b-c$ in Eq. (4)) and also for the cases where the coupler is in-line with the input link (just interchange $\phi$ and $\psi$ ) instead of the output link. Similarly, one can address a mixed function generation synthesis problem with the positions where two of the positions are the folded and extended dead-center position and the remaining position is given as a correlation of crank angles. The given two dead-center positions might be specified as one for the input link and one for the output link as well. 
Or else, one or two of the positions might be specified for a position where the input or the output link is in-line with the fixed link (this condition is usually evaluated for maximum deviation of the transmission angle from $90^{\circ}$ ). Furthermore, for the cases where the exact angle of the input or the output angles is not important, but a desired angular displacement of the links is specified (as in a typical function generation problem), it is possible to introduce initial input and or output angle values as additional design parameters and design for four or five positions (See for ex. [2]). But in this case the nonlinearity of the problem increases and there may be no analytical solution. For instance, for given extended/folded dead center output angles and correlation of crank angles for a general configurations results in a sixth order univariate polynomial, which has no analytical solution but can be rapidly solved using a simple root finding algorithm. For more than 5 positions analytical optimization methods such as least square or Chebyshev approximation methods or numerical optimization methods might be utilized. A mixed function generation synthesis problem may be formulated for all these cases, but these formulations are kept out-off scope of this study and left for further studies for potential applications.

\section{Acknowledgment}

The authors thank to Prof. Eres Söylemez for his comments and suggestions.

\section{References}

1. Söylemez, E.: Mechanisms. 5th edn. METU Press, Ankara (2018).

2. Hartenberg, R. S., Denavit, J.: Kinematic Synthesis of Linkages. McGraw-Hill, New York (1964).

3. Sandor, G. N., Erdman A. G., Advanced Mechanism Design: Analysis and Synthesis Vol. II. Prentice Hall, New Jersey (1984).

4. McCarthy, J. M., Soh, G. S.: Geometric Design of Linkages. $2^{\text {nd }}$ edn. Springer, New York (2011).

5. Hall, A. S.: Kinematics and Linkage Design. Prentice-Hall, Englewood Cliffs (1961).

6. Norton, R. L.: Design of Machinery. 6th edn. McGraw-Hill, Boston (2019).

7. Mallik, A. K., Ghosh, A., Dittrich, G.: Kinematic Analysis and Synthesis of Mechanisms. CRC Press, Boca Raton (1994).

8. Pennestrì, E., Valentini, P. P.: A review of simple analytical methods for the kinematic synthesis of four-bar and slider-crank function generators for two and three prescribed finite positions. In: Buletin Stiintific Seria Mecanica Aplicata, pp. 128-143. University of Pitesti, Pitești, (2009).

9. Freudenstein, F.: An analytical approach to the design of four-link mechanisms. Trans. ASME 76, 483-492 (1954). 